Supporting information

\title{
Investigation of Hot Carrier Cooling Dynamics in the Monolayer $\mathrm{MoS}_{2}$
}

Wenyan Wang ${ }^{1}$, Ning Sui ${ }^{1}$, Xiaochun Chi ${ }^{1}$, Zhihui Kang ${ }^{1}$, Qiang Zhou ${ }^{3}, \mathrm{Li} \mathrm{Li}^{4}$, Hanzhuang Zhang ${ }^{1}$, Jianbo Gao ${ }^{2 *}$, and Yinghui Wang ${ }^{1 *}$

${ }^{1}$ Femtosecond Laser laboratory, Key Laboratory of Physics and Technology for Advanced Batteries, College of Physics, Jilin University, Changchun 130012, China.

${ }^{2}$ Ultrafast Photophysics of Quantum Devices Laboratory, Department of Physics and Astronomy, Clemson University, South Carolina, 29634, USA

${ }^{3}$ State Key Laboratory of Superhard Materials, College of Physics, Jilin University, Changchun 130012, China.

${ }^{4}$ College of Physics and Optoelectronic Engineering, Harbin Engineering University, Harbin, 150001, China 
Supplementary Figure 1. SEM of M-MoS 2 .

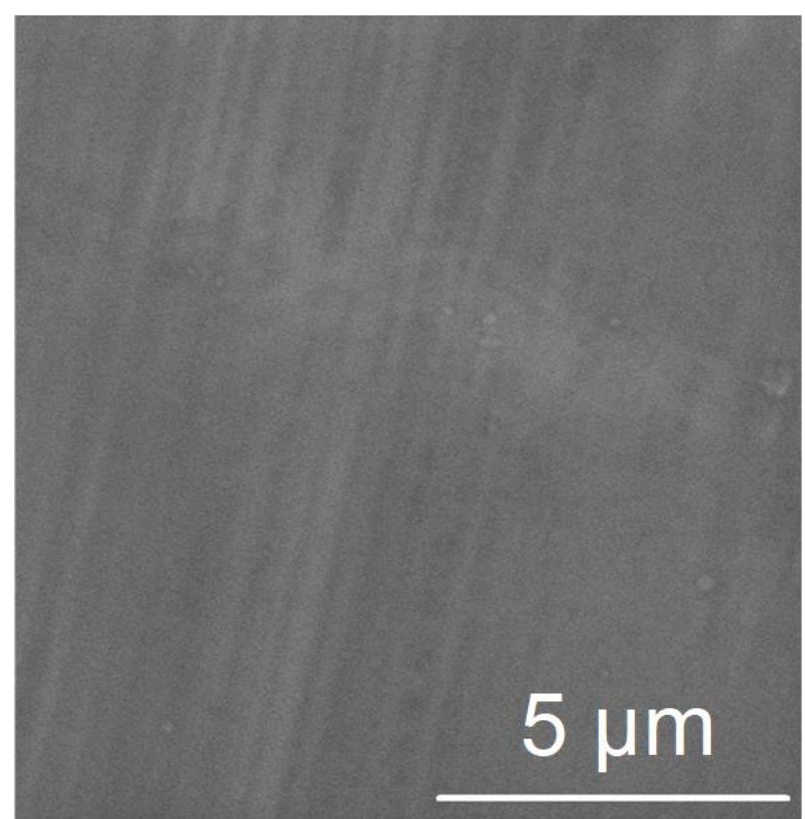


Supplementary Figure 2. The 2D TA spectra of $\mathrm{M}-\mathrm{MoS}_{2}$ with the excitation photon energy of (a) $1.88 \mathrm{eV}$, (b) 2.07 and (c) $3.10 \mathrm{eV}$, the corresponding absorbed photon flux is $1.03 \times 10^{13}$ photon $\mathrm{cm}^{-2}$.

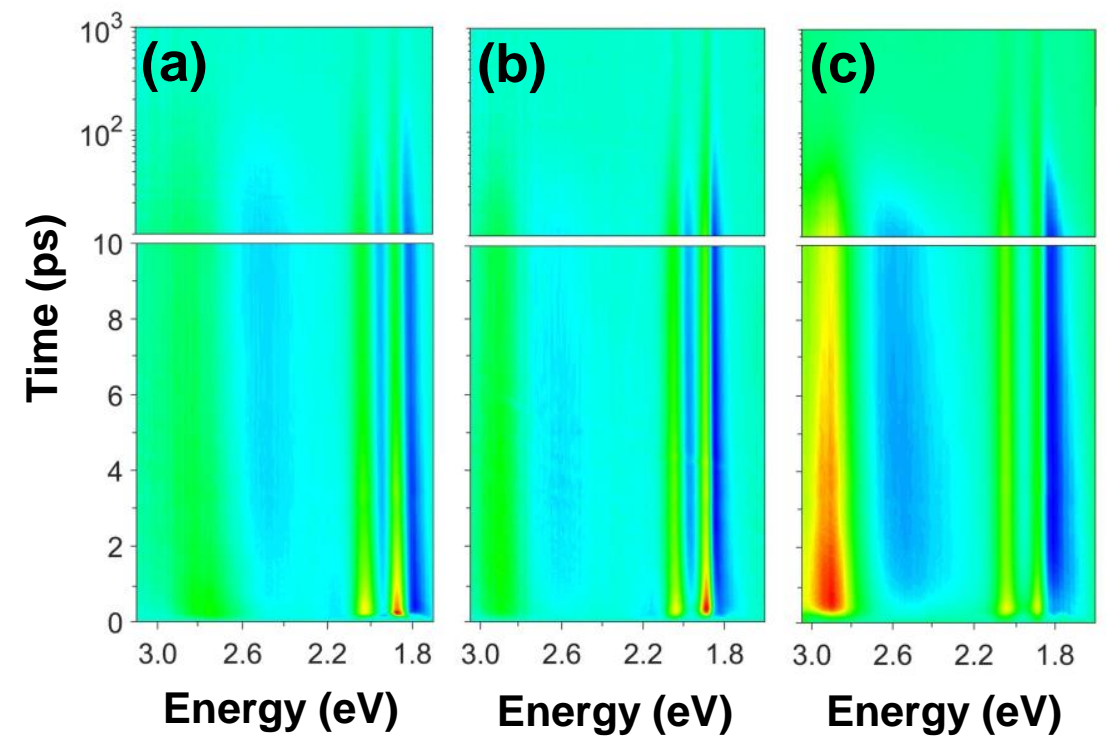


Supplementary Figure 3. Excitation photon energy-dependent TA spectra of $\mathrm{M}-\mathrm{MoS}_{2}$ with absorbed photon flux of $5.15 \times 10^{12}$ photon $\mathrm{cm}^{-2}$ at the delay time when C-PIA amplitude rises to the maximum.

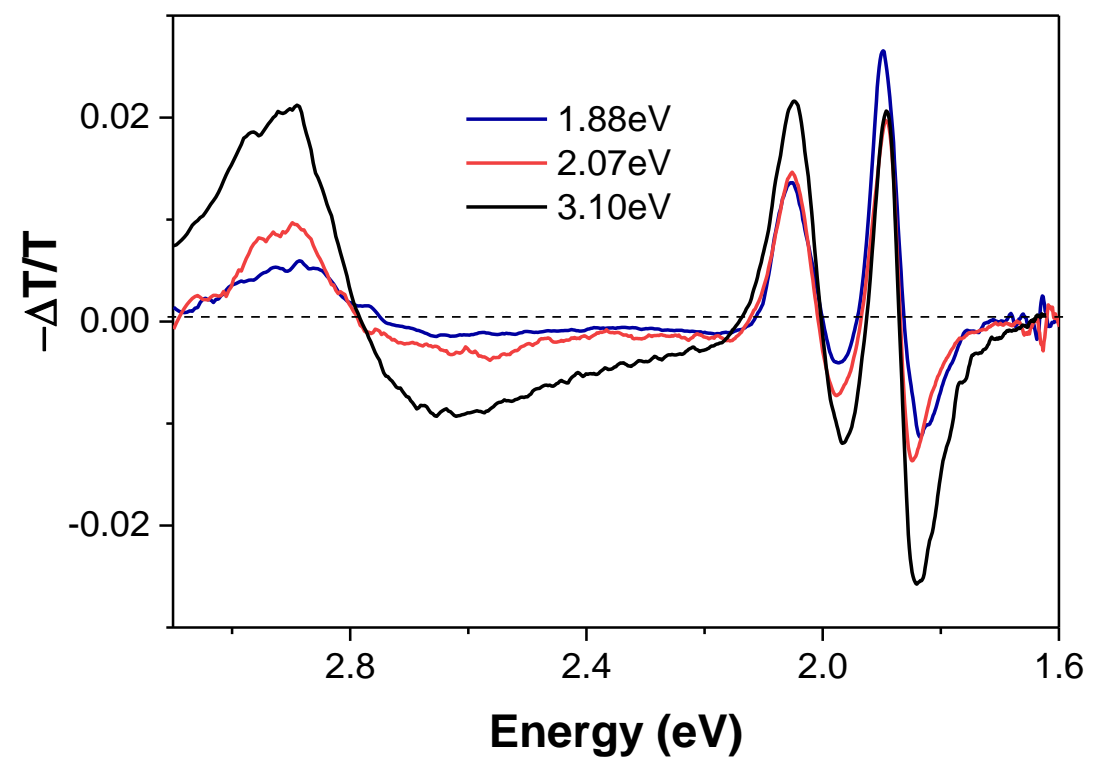


Supplementary Figure 4. Absorbed photon flux-dependent TA spectra of M-MoS with excitation photon energy of $1.88 \mathrm{eV}$ at the delay time when C-PIA amplitude rises to the maximum.

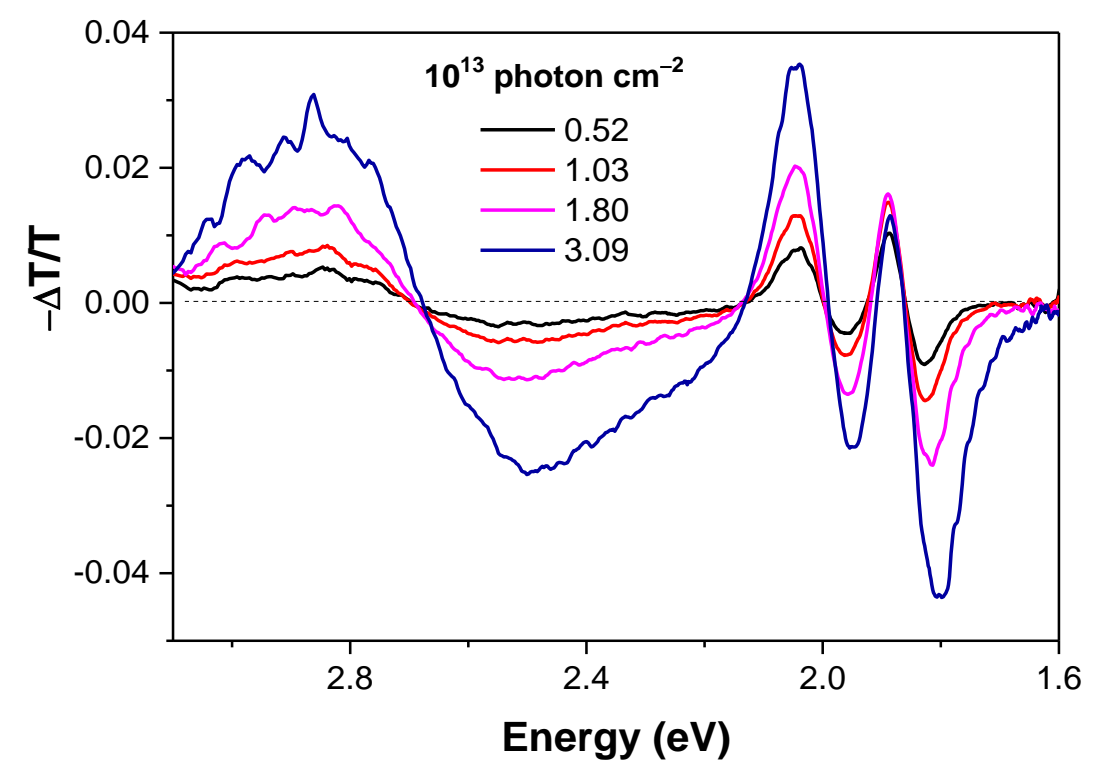


Supplementary Figure 5. Absorbed photon energy-dependent C-PIA curves of M$\mathrm{MoS}_{2}$ with the excitation photon energy of (a) $2.07 \mathrm{eV}$ and (b) $3.10 \mathrm{eV}$.

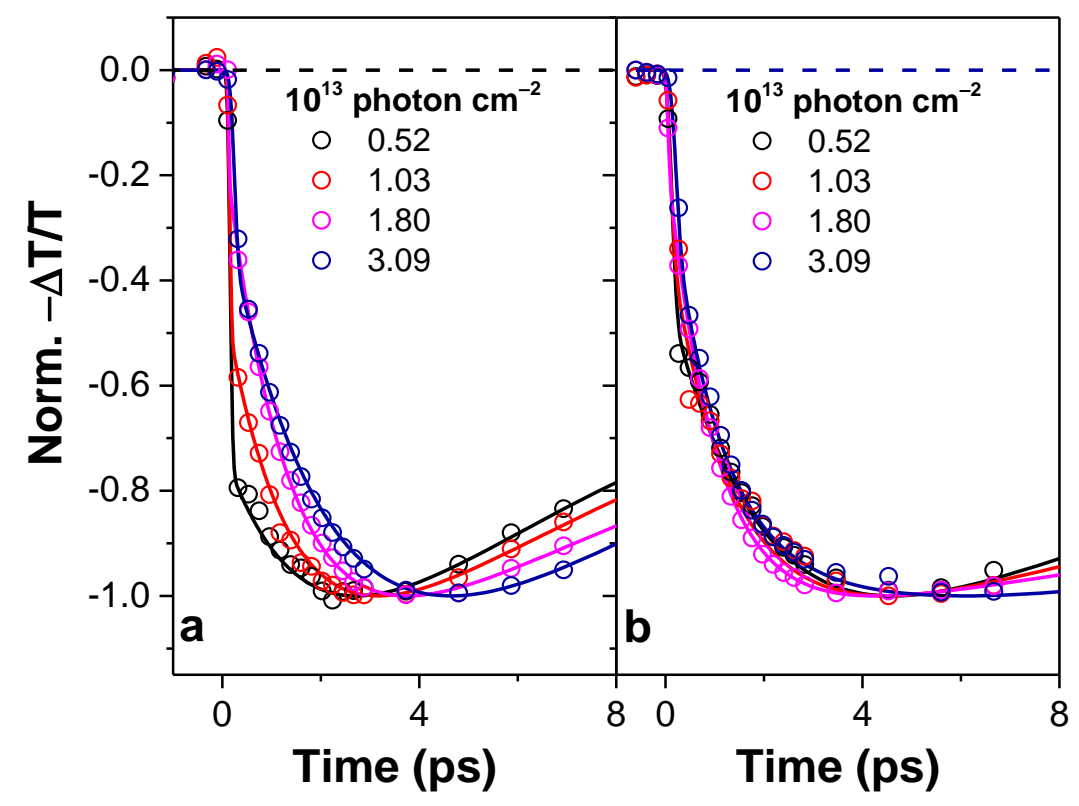


Supplementary Figure 6. Absorbed photon flux-dependent C-PB curves of M-MoS with the excitation photon energy of $3.10 \mathrm{eV}$.

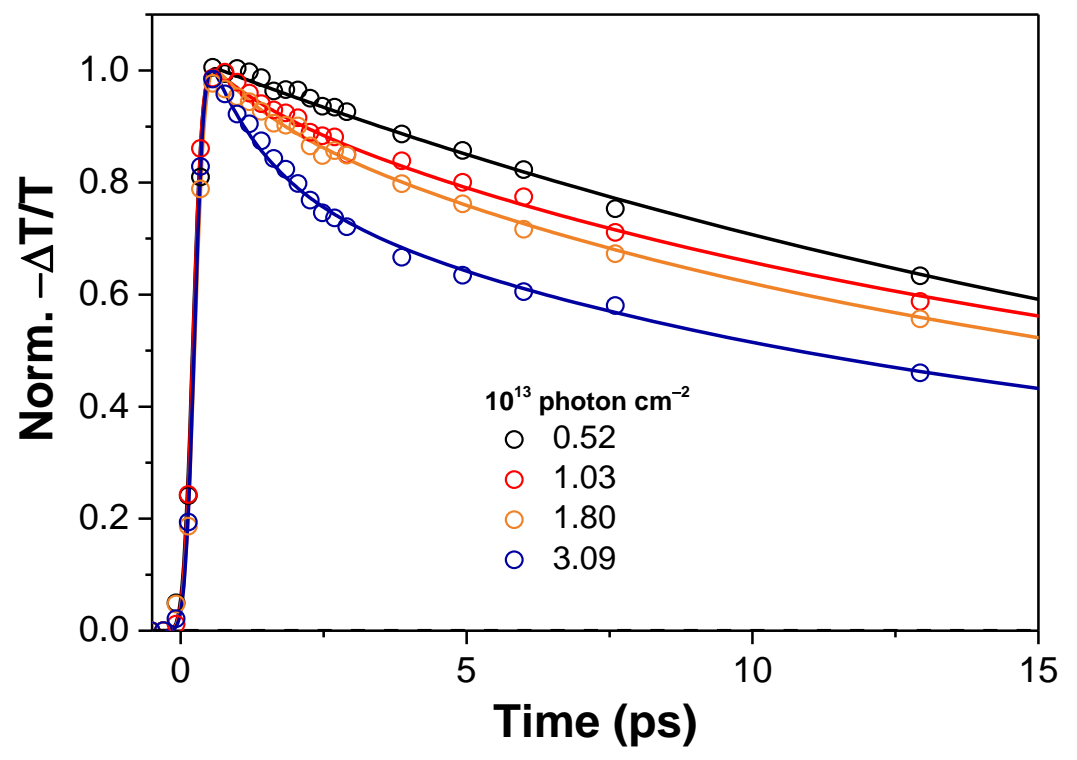


Supplementary Figure 7. Absorbed photon flux-dependent TA spectra of M-MoS with excitation photon energy of $2.07 \mathrm{eV}$ at the delay time, when C-PIA amplitude rises to the maximum.

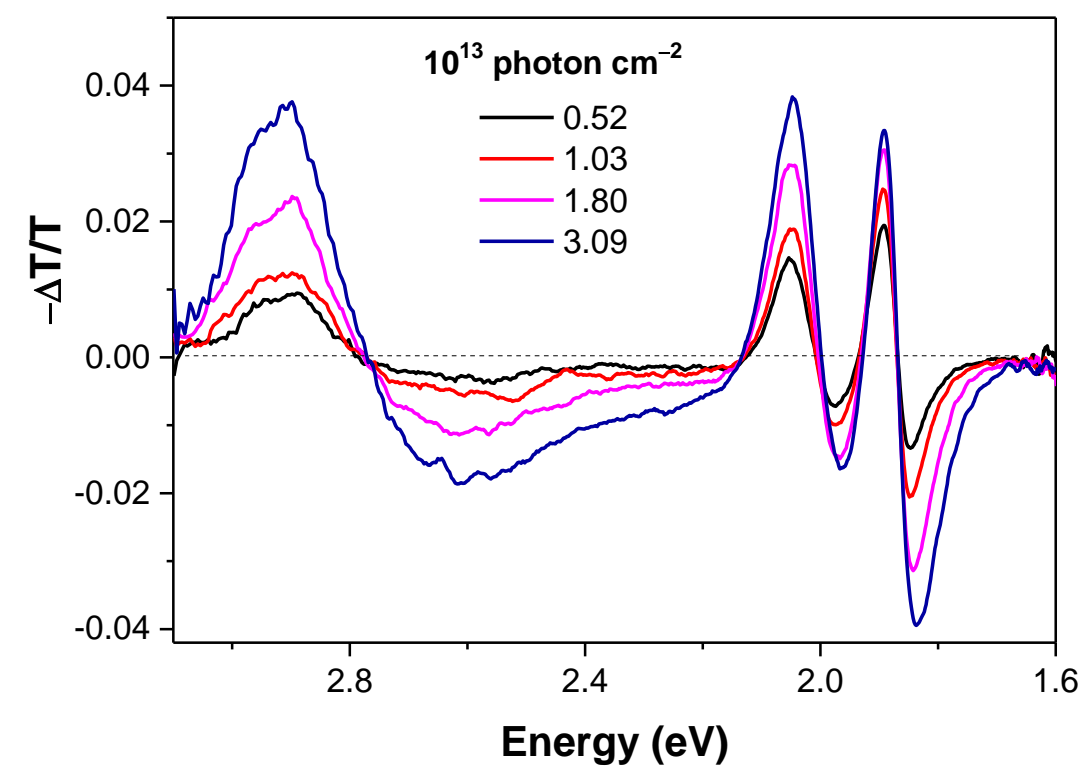


Supplementary Figure 8. (a) Absorbed photon flux-dependent absolute value of initial A-PB signal and that of C-PIA signal when it reaches to the maximum with the excitation photon energy of $1.88 \mathrm{eV}$; (b) Absorbed photon flux-dependent absolute value of initial C-PB signal and that of C-PIA signal when it reaches to the maximum with the excitation photon energy of $3.10 \mathrm{eV}$.

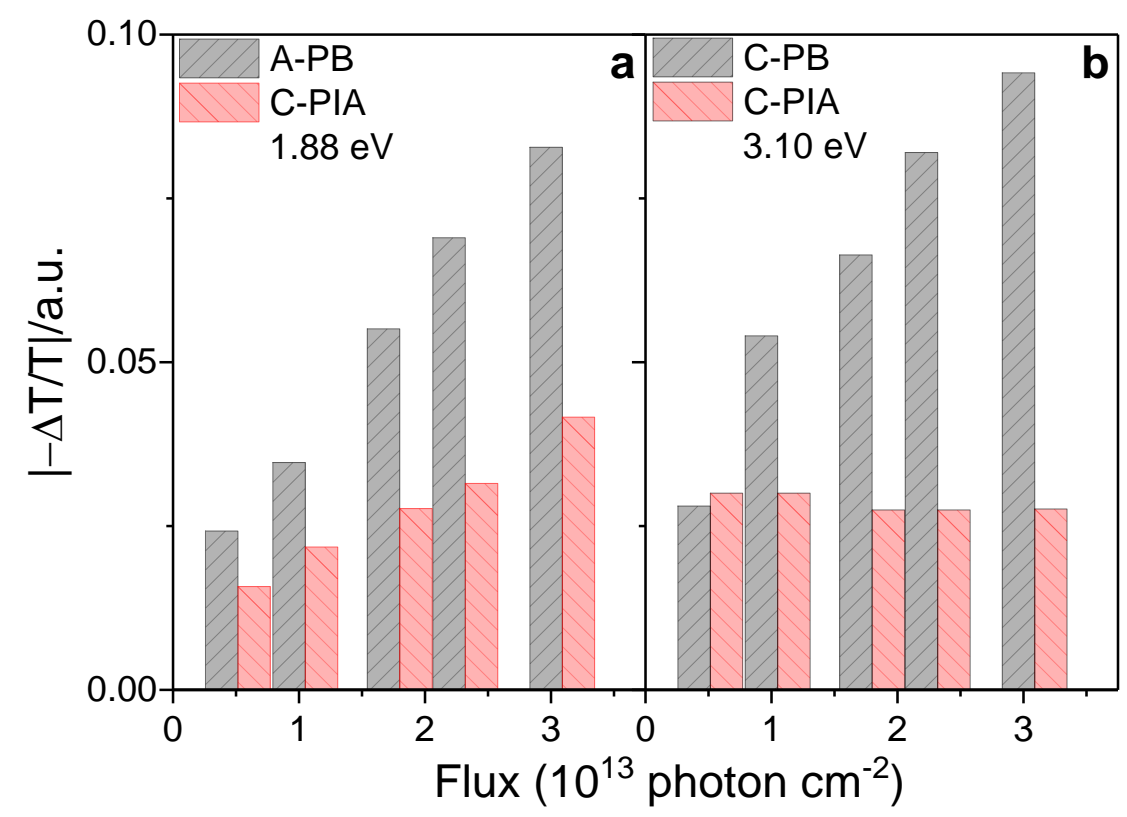


Supplementary Figure 9. Absorbed photon flux-dependent TA spectra of M-MoS with excitation photon energy of $3.10 \mathrm{eV}$ at the delay time when C-PIA amplitude rises to the maximum.

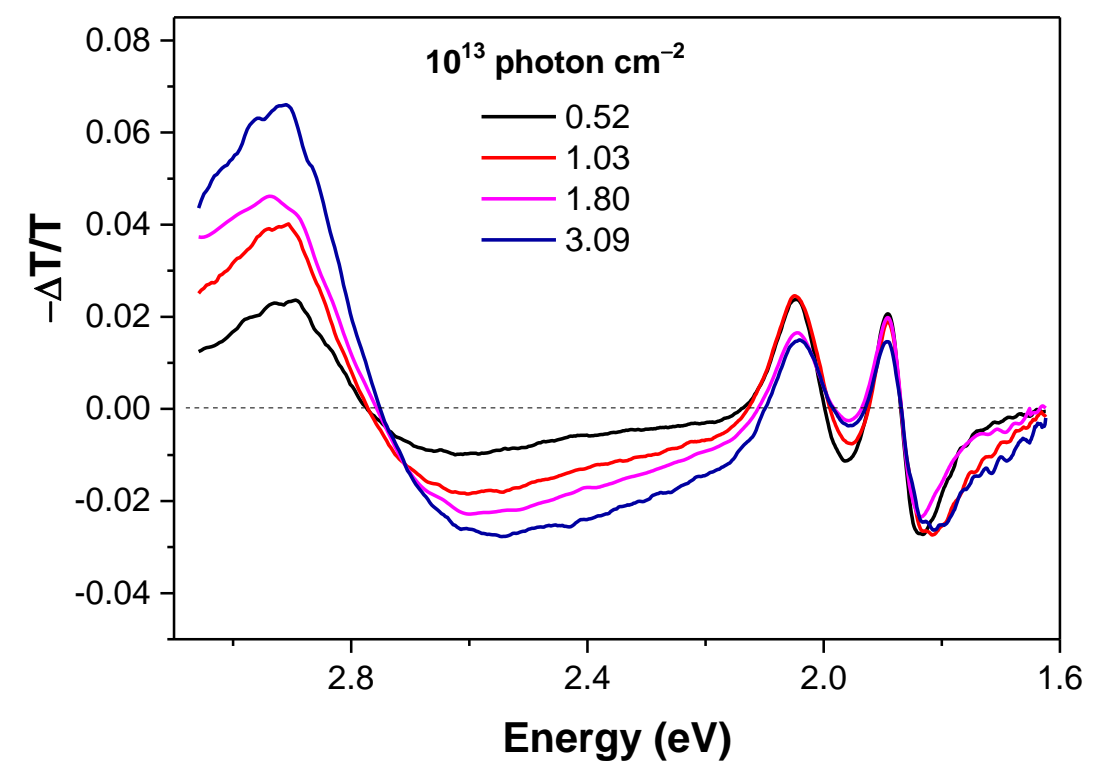


Supplementary Figure 10. A/B-PIA curves of $\mathrm{M}-\mathrm{MoS}_{2}$ with different excitation photon energy, where the absorbed photon flux is (a) $1.80 \times 10^{13}$ and (b) $5.15 \times 10^{12}$ photon $\mathrm{cm}^{-2}$, respectively. Inset: The corresponding cooling time $\left(\tau_{\mathrm{HC}-\mathrm{C}}\right)$ of hot carriers at different excitation photon energy.
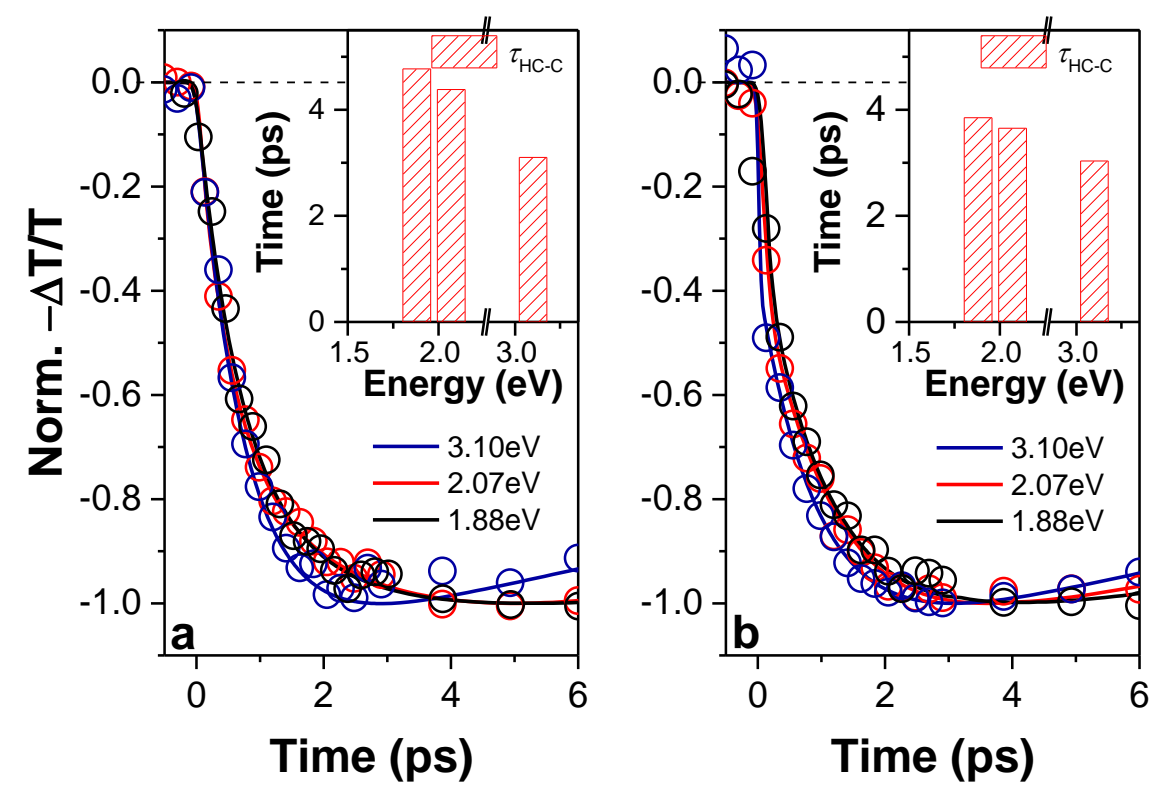
Supplementary Figure 11. Energy diagram of hot carriers in A/B-X and C-X affected by the excitation photon energy and absorbed photon flux. The excitation photon energy is (a) $1.88 \mathrm{eV}$, (b) $2.07 \mathrm{eV}$ and (c) $3.10 \mathrm{eV}$. HPB: Hot phonon bottleneck; PE: phonon emission; RP: reabsorption of phonon.
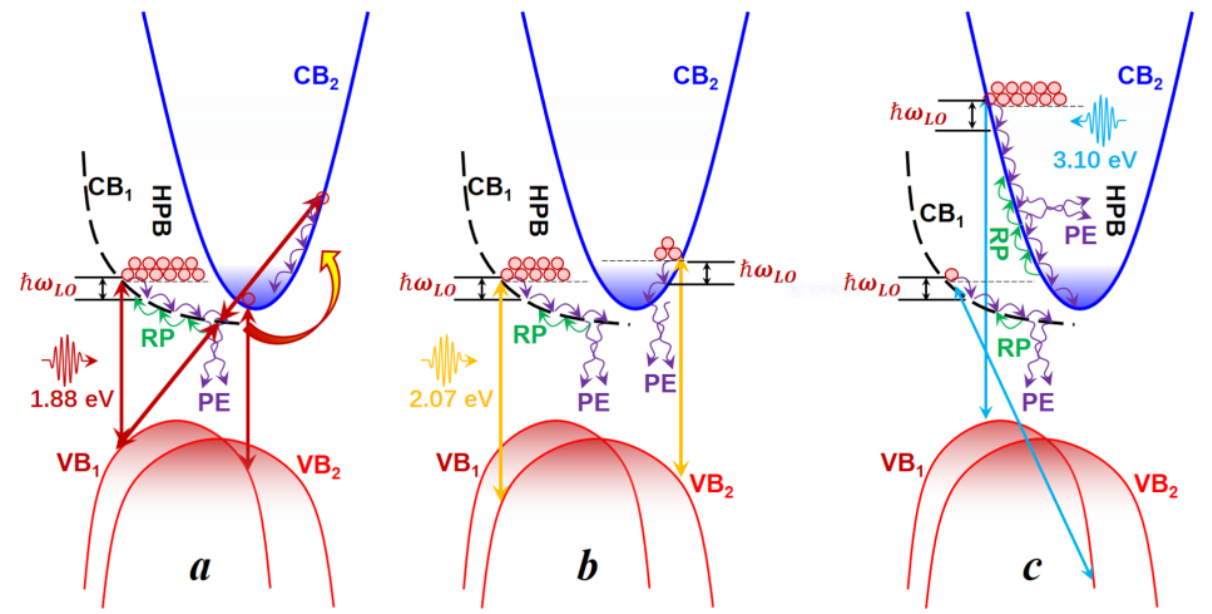
Supplementary Figure 12. Absorbed photon flux-dependent $\tau_{\mathrm{HC}-\mathrm{C}}$ of hot carriers in $\mathrm{A} / \mathrm{B}-\mathrm{X}$ of $\mathrm{M}-\mathrm{MoS}_{2}$ with the excitation photon energy of $1.88,2.07$ and $3.10 \mathrm{eV}$.

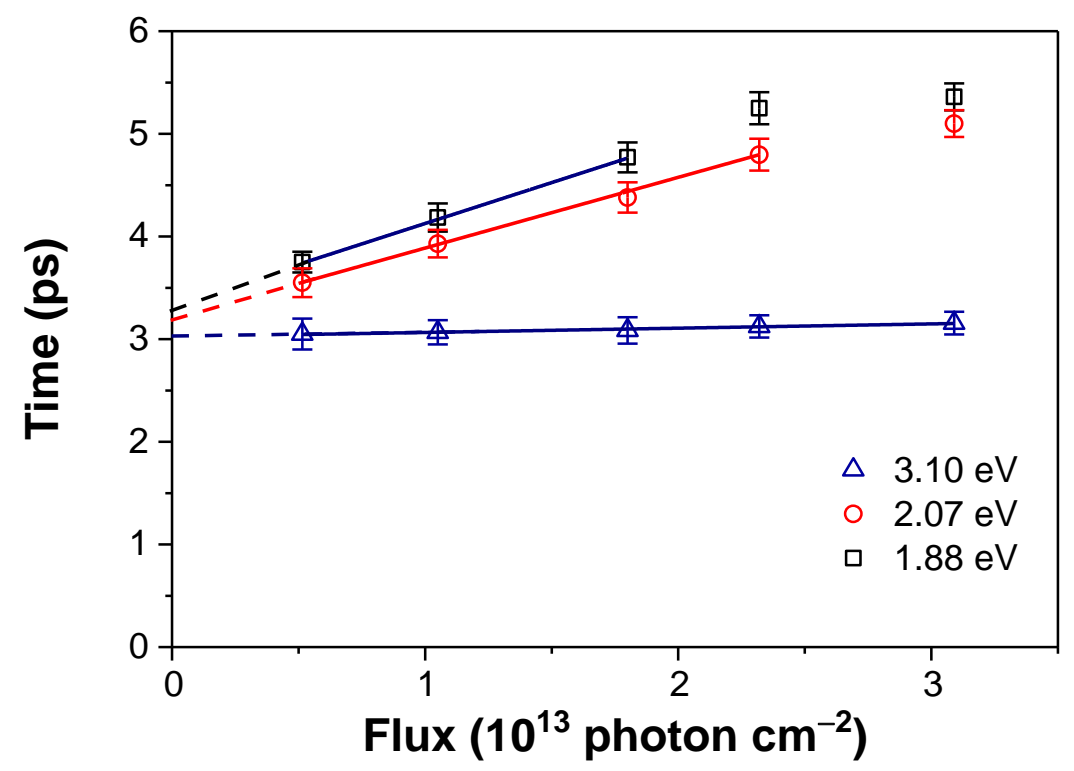


Supplementary Figure 13. Absorbed photon flux-dependent A/B-PIA curves of M$\mathrm{MoS}_{2}$ with the excitation photon energy of (a) 1.88 , (b) 2.07 and (c) $3.10 \mathrm{eV}$.

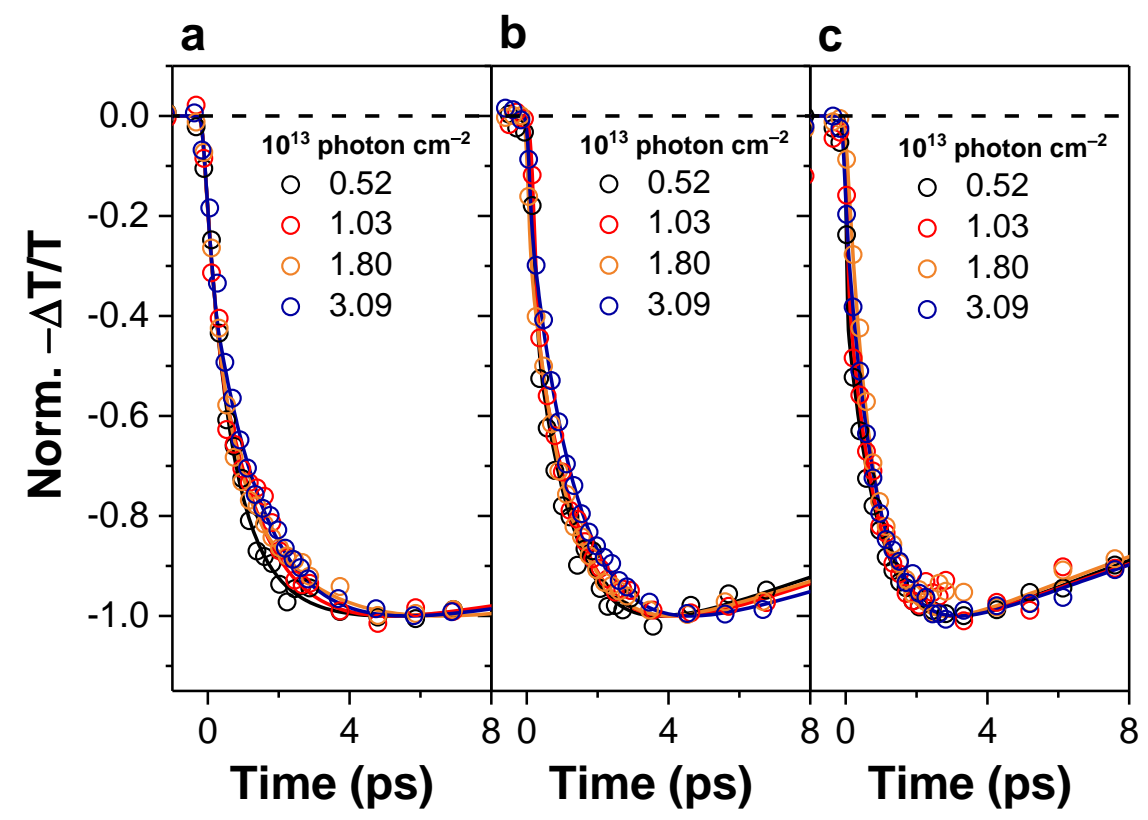


Supplementary Figure 14. The pump laser spectrum with the photon energy of 1.88, 2.07 and $3.10 \mathrm{eV}$.

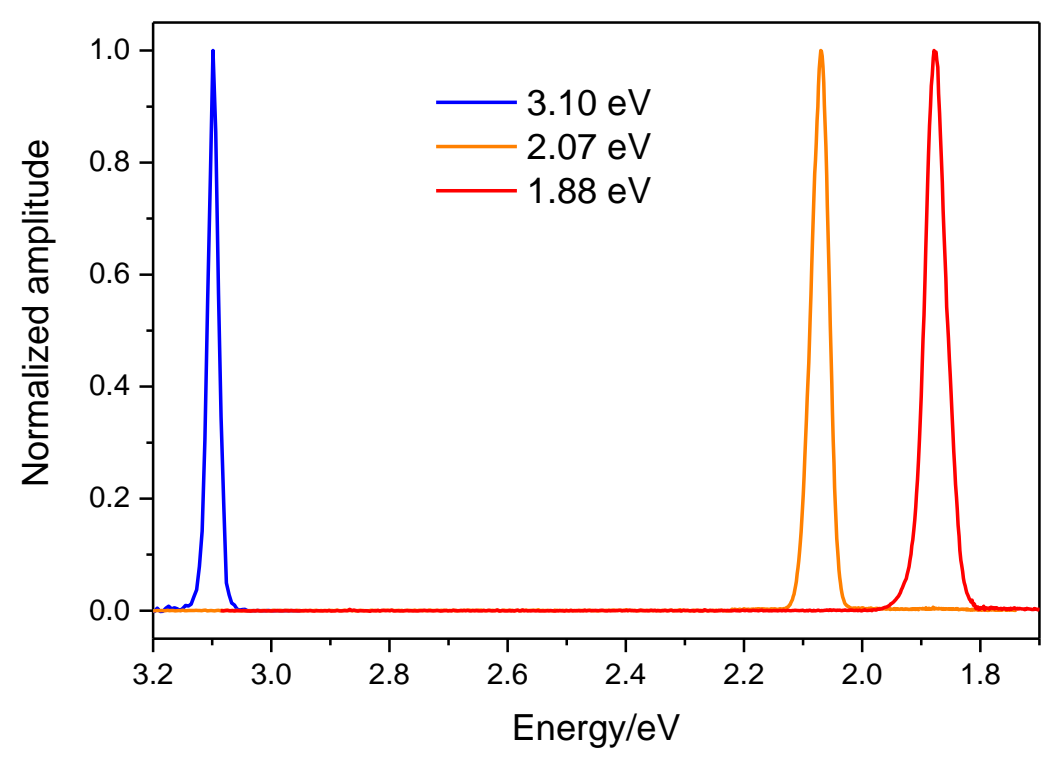




\section{Supplementary Notes}

Supplementary Note 1: Distribution of carriers in monolayer $\mathrm{MoS}_{2}$ manipulated by excitation photon energy.

$\mathrm{M}-\mathrm{MoS}_{2}$ owns rich structure of energy band, as illustrated in Figure 1b. For a given excitation photon energy, it can distribute carriers in different electronic states simultaneously. The corresponding TA spectra in Figure 1d exhibits the photobleaching ${ }^{1}$ signal of the A-X, B-X and C-X, which can also confirm it. Note that the carrier in $\mathrm{C}-\mathrm{X}$ is located in the bottom of $\mathrm{CB}_{2}$ as illustrated in Figure 4c. As the excitation photon energy increases to $2.07 \mathrm{eV}$, the photo-generated carriers can also be distributed in A-X, B-X and C-X simultaneously. In this situation, the energy state position of photo-generated carrier in $\mathrm{C}-\mathrm{X}$ is apparently higher in comparison with the bottom of $\mathrm{CB}_{2}$. If the excitation photon energy keeps increasing to $3.1 \mathrm{eV}$, the energy state position of photo-generated carrier in $\mathrm{C}-\mathrm{X}$ can be much higher than the bottom of $\mathrm{CB}_{2}$. In a word, the excitation laser pulse can distribute the carriers in A-X, B-X and C$\mathrm{X}$ simultaneously, if the excitation photon energy is in the region of absorption band. Meanwhile, the energy state position of photo-generated carrier in $\mathrm{C}-\mathrm{X}$ rises with increasement of excitation photon energy.

\section{Supplementary Note 2: Calculation and fitting of dynamic curve.}

The C-PIA curve was fitted following a three-exponential function as follow ${ }^{2}$ :

$$
I=A_{1} * \mathrm{e}^{-\left(\frac{t}{\tau_{1}}\right)}+A_{2} * \mathrm{e}^{-\left(\frac{t}{\tau_{2}}\right)}+A_{3} * \mathrm{e}^{-\left(\frac{t}{\tau_{3}}\right)}
$$

Here $A_{1}, A_{2}$ and $A_{3}$ represent the population of each component. And the $\tau_{1}$ represent lifetime of the raise component contributed by hot carrier $\left(\tau_{\mathrm{HC}-\mathrm{C}}\right)$.

Supplementary Note 3: Correlation between excitation photon energy and absorbed photon flux in A/B-X.

Figure S12 shows the absorbed photon flux-dependent cooling time of hot carrier in A/B-X excited by different excitation photon energy. The $\tau_{\text {HC-C }}$ of A/B-X approximately linearly increases with absorbed photon flux and then gradually approach a constant as 
the excitation photon energy of $1.88 \mathrm{eV}$ and $2.07 \mathrm{eV}$. Apparently, the hot carrier characteristics in A/B-X at different absorbed photon flux (as seen in Figure S12) are different from that in $\mathrm{C}-\mathrm{X}$ as seen in Figure $4 \mathrm{a}$, which is attributed to the variance of hot phonon bottleneck induced by different carrier density. When the excitation photon energy is $1.88 \mathrm{eV}$, the photon can directly induce the hot carriers in $\mathrm{A} / \mathrm{B}-\mathrm{X}$, whose cooling time can prolong with the absorbed photon flux as illustrated in Figure S11a. In this situation, the most photo-generated carriers are distributed in the $\mathrm{CB}_{1}$ and only a little part of carriers can be distributed in $\mathrm{CB}_{2}$, whose dynamic process is given detailed in the main body. After the absorbed photon flux is closed to $1.80 \times 10^{13}$ photon $\mathrm{cm}^{-2}$, the Auger recombination occurs as seen in Figure 3c. In this situation, some carriers jumped into high energy state through Auger reheating, such as $\mathrm{C}-\mathrm{X}$, and some carriers go back to the ground state owing to the recombination. Finally, the carrier density in $\mathrm{A} / \mathrm{B}-\mathrm{X}$ increases a little after the absorbed photon flux increases to $1.80 \times 10^{13}$ photon $\mathrm{cm}^{-2}$. In this situation, the hot phonon bottleneck effect of $\mathrm{CB}_{1}$ doesn't enhance apparently and also can't further prolong the cooling lifetime of hot carriers in A/B-X (as seen in Figure S12). As the excitation photon energy increases to $2.07 \mathrm{eV}$, some of generated carriers directly into $\mathrm{C}-\mathrm{X}$ and other carriers remains in the $\mathrm{A} / \mathrm{B}-\mathrm{X}$, since $\mathrm{A}-\mathrm{X}$ and B-X share the same conduction band (as illustrated in Figure S11b). In this situation, the carrier density of $\mathrm{A} / \mathrm{B}-\mathrm{X}$ at the excitation photon energy of $2.07 \mathrm{eV}$ is lower than that at the excitation photon energy of $1.88 \mathrm{eV}$, which weakens the hot phonon bottleneck effect at the same time. Therefore the cooling time of hot carrier in A/B-X at the excitation photon energy of $2.07 \mathrm{eV}$ is lower than that at the excitation photon energy of $1.88 \mathrm{eV}$ (as seen in Figure S12). As the excitation photon energy is fixed at $3.10 \mathrm{eV}$, the $\tau_{\mathrm{HC}-\mathrm{C}}$ in $\mathrm{A} / \mathrm{B}-\mathrm{X}$ increases a little with the increasement of absorbed photon flux, since the most of carriers is distributed on the $\mathrm{C}-\mathrm{X}$ and only a little part of carriers are located at A/B-X (as illustrated in Figure S11c). Even though the absorbed photon flux increases, the carrier density in A/B-X changes a little, which can't prolong the cooling time of hot carriers through enhancing the hot phonon bottleneck effect. In a word, the complex energy structure of $\mathrm{M}-\mathrm{MoS}_{2}$ cause that the photo-generated carriers 
can distribute in $\mathrm{A} / \mathrm{B}-\mathrm{X}$ and $\mathrm{C}-\mathrm{X}$ simultaneously (as mentioned in Supplementary Note 1). The proportion of carriers in $\mathrm{C}-\mathrm{X}$ increases, when the excitation photon energy increases from 1.88 to $3.1 \mathrm{eV}$. However, that in $\mathrm{A} / \mathrm{B}-\mathrm{X}$ is on the contrast. Therefore, the hot carrier cooling process in $\mathrm{C}-\mathrm{X}$ is different from that in $\mathrm{A} / \mathrm{B}-\mathrm{X}$ (as seen in Figure 4a and Figure S12) as explained above. But the Auger recombination and hot phonon bottleneck together play an important role in the hot carrier cooling dynamics.

\section{Supplementary Note 4: Calculation of absorbed photon flux.}

The absorbed photon flux $N$ is calculated with the following formulas:

$$
\begin{gathered}
N=\frac{N_{0} x}{\pi r^{2}} \\
N_{0}=\frac{P t}{h v} \\
t=\frac{1}{f} \\
v=\frac{c}{\lambda} \\
x=\left(1-10^{-O D}\right) \times 100 \%
\end{gathered}
$$

where $N_{\boldsymbol{o}}$ is the total photon number per pump laser pulse, $\boldsymbol{x}$ is the $\mathrm{M}-\mathrm{MoS}_{2}$ absorption percentage at the pump wavelength, $\boldsymbol{r}$ is the pump laser radius $(150 \mu \mathrm{m}), \boldsymbol{P}$ is the average power of pump laser, $\boldsymbol{h}$ is the Planck constant, $\boldsymbol{v}$ is the pump laser frequency, $\boldsymbol{f}$ is pump laser repetition frequency $(250 \mathrm{~Hz}), \boldsymbol{c}$ is velocity of light and $\boldsymbol{O D}$ is optical density of $\mathrm{M}-\mathrm{MoS}_{2}$ at the pump laser wavelength.

\section{Supplementary Note 5: Pump laser characteristics of different photon energy.}

The pump laser is generated via an optical parameter amplifier (Coherent, TOPAS) and the output wavelength is reliable and can be controlled by software. In addition, to make sure the wavelength of pump laser is clean without any unexpected wavelength, we have also measured the pump laser spectra. The normalized pump laser spectra with photon energy of 1.88, 2.07 and $3.10 \mathrm{eV}$ are exhibited in Figure S13. It displays a clean single peak without any unexpected signal. 


\section{Supplementary Note 6: Selection of absorbed photon flux.}

In 2D TMDCs, the exciton, biexciton, trion and free carrier can be generated simultaneously after photo excitation ${ }^{3}$. Thus, it is not accurate to calculate the carrier density from the pump laser power. For this reason, we use the absorbed photon flux to describe the experimental condition and make sure the photon number that absorbed by the $\mathrm{M}-\mathrm{MoS}_{2}$ can be estimated when the excitation photon energy changed from 1.88 to $3.10 \mathrm{eV}$. 


\section{References}

(1) Shi, H.; Yan, R.; Bertolazzi, S.; Brivio, J.; Gao, B.; Kis, A.; Jena, D.; Xing, H. G.; Huang, L., Exciton dynamics in suspended monolayer and few-layer $\mathrm{MoS}_{2} 2 \mathrm{D}$ crystals. ACS Nano 2013, 7 (2), 1072-1080.

(2) Robles, F. E.; Wilson, J. W.; Fischer, M. C.; Warren, W. S., Phasor analysis for nonlinear pump-probe microscopy. Opt. Express 2012, 20 (15), 17082-17092.

(3) You, Y.; Zhang, X.-X.; Berkelbach, T. C.; Hybertsen, M. S.; Reichman, D. R.; Heinz, T. F., Observation of biexcitons in monolayer WSe 2. Nat. Phys. 2015, 11 (6), 477. 